Research Article

\title{
Effects of Nanoparticles on the Antipullout Strength between the Reinforcement and Cement Mortar
}

\author{
Yupeng Shen,, ${ }^{1,2}$ Peng Jing $\mathbb{D}^{3}{ }^{3}$ Wensheng Yao, ${ }^{4}$ Tianxiao Tang, ${ }^{1}$ Dandan Cao, ${ }^{3}$ \\ Hongshang Peng, ${ }^{5}$ and Yaqiong Wang ${ }^{6}$ \\ ${ }^{1}$ School of Civil Engineering, Beijing Jiaotong University, Beijing 100044, China \\ ${ }^{2}$ Beijing Key Laboratory of Track Engineering, Beijing Jiaotong University, Beijing 100044, China \\ ${ }^{3}$ College of Metropolitan Transportation, Beijing University of Technology, Beijing 100124, China \\ ${ }^{4}$ Liaoning Nonferrous Geological Exploration and Research Institute Co., Shenyang 110013, China \\ ${ }^{5}$ College of Science, Minzu University of China, Beijing 100081, China \\ ${ }^{6}$ Chang'an University, Shaanxi Provincial Major Laboratory for Highway Bridge \& Tunnel, Xi'an, Shaanxi 710064, China
}

Correspondence should be addressed to Peng Jing; peng.jing@bjut.edu.cn

Received 4 May 2020; Revised 12 July 2020; Accepted 14 July 2020; Published 30 July 2020

Academic Editor: Yuqing Zhang

Copyright (C) 2020 Yupeng Shen et al. This is an open access article distributed under the Creative Commons Attribution License, which permits unrestricted use, distribution, and reproduction in any medium, provided the original work is properly cited.

Recently, the use of nanomaterials (i.e., Nano-Silica (NS) and Nano-SiC(NC)) to improve the mechanical properties and durability performances of cement-based materials has received considerable attention. This work presents the effects of nanoparticles (NS), the specimen size, and the water-reducing agent on the antipullout strength between the reinforcement and cement mortar on the basis of the laboratory antipullout tests. The mechanism is, then, validated by the microscopic analysis with Scanning Electron Microscopy (SEM). Results show that NS can accelerate the hydration reaction of cement, produce more Calcium Silicate Hydrate (CSH) gel, and increase the density of the cement mortar, thereby improving the microstructure and antipullout strength. The moderate NS (1.0\%) is recommended to improve antipullout between the reinforcement and cement mortar. Besides, the specimen size and water-reducing agent have an obvious influence on the antipullout strength. These results would provide guidance on the design or construction of the cement mortar with reinforcement.

\section{Introduction}

Nowadays, slope stability analysis is becoming increasingly important in road design and construction. Regarding this matter, bolting, being an effective way of strengthening the slope, transfers the sliding force from unstable soil to stable surrounding rocks. However, some engineering accidents have still occurred because of the bolt splitting out from grouts in a bolt support system [1-3].

The use of both chemically active and inert mineral admixtures to cement-based materials has grown during the last few decades due to their technical advantages (increase in strength and durability) and environmental consideration (associated to the reduction in cement consumption) [4].

One of those is adding fine Nano-Silica (NS) to improve compatibility between two materials with different hydrophilic properties and to ameliorate the bonding properties between cement and the steel-bar $[5,6]$. The most reported effect of NS is its influence on the mechanical properties of concrete and mortars. Adding NS increases density, decreases porosity, and increases the bond between the cement matrix and aggregates $[5,7-15]$. The presence of NS also produces concrete that exhibits higher compressive and flexural strength $[6,12,16,17]$. Cement mortar-added NS particles showed that nanoscale $\mathrm{SiO}_{2}$ behaves as a filling to improve the microstructure and as an activator to promote the pozzolanic reaction.

In the meanwhile, to ensure that fresh concrete has enough fluidity and hardened concrete has sufficient strength and durability, a certain amount of water-reducing agents are typically added. Lacking sulfate of soluble alkali, cement will absorb more naphthalene-based water-reducing agent molecules, and as a result, high absorption of the 
naphthalene-based water-reducing agent leads to lower initial slump of the mixture and more significant timecorrelated slump loss $[18,19]$. With the mixing of NS, the dry shrinkage rate of cement concrete will increase. Also, by adding water-reducing agents, the rheological properties of concrete can be improved, and the shrinkage rate of NS cement concrete can be controlled [20-24].

Based on the literatures, the improvement of the gripping force between the cement mortar and reinforcement by adding NS has been rarely studied, especially the effect of the nanoparticle amount on the gripping force.

In this context, this paper initially presents effects of NS and the water-reducing agent and size effect on the antipullout strength between the reinforcement and cement mortar based on the laboratory antipullout tests. The mechanism is, then, studied by the microscopic analysis with Scanning Electron Microscopy (SEM).

\section{Experimental Program}

2.1. Materials Properties and Mixture Proportions. Portland cement type is PC32.5R and the main chemical composition are presented in Table 1. Reinforced bar is HRB 335 in a diameter of $16 \mathrm{~mm}$. Tetraethoxysilane (TEOS, 98\%) is obtained from Jiaxing Sicheng Chemicals Co., Ltd. (China). Ethanol (99.5\%) and ammonia (25-28\%) are from Lanyi Chemical Co., Ltd. (China). The UNF-5 naphthalenebased water-reducing agent is compounded by the $\beta$-naphthalene sulfonate which is obtained from Kamile-su Chemical Co., Ltd. (China).

The synthesis of NS particles involves several steps: first, in a $500 \mathrm{ml}$ three-necked flask, $57.4 \mathrm{ml}$ of TEOS is added dropwise to $100 \mathrm{ml}$ of aqueous solution under mild magnetic stirring, which contains ethanol $(34.5 \mathrm{ml})$ and ammonia $(5.74 \mathrm{ml})$. After reacting for $6 \mathrm{~h}$ at $30^{\circ} \mathrm{C}$ under a water bath, the products are collected by centrifugation and washed with absolute ethanol. The products are, then, dried at $120^{\circ} \mathrm{C}$ for $3 \mathrm{~h}$, and NS particles are obtained.

Eight different set of mixtures on the basis of an ordinary Portland cement mortar are considered for this test, as listed in Table 2, consisting of M1 without NS, M2 containing $0.5 \%$ NS, M3 containing $1.0 \%$ NS, M4 containing $1.5 \%$ NS, M5 containing $1.0 \%$ water-reducing agent without NS, M6 containing $1.0 \%$ water-reducing agent and $0.5 \%$ NS, M7 containing $1.0 \%$ water-reducing agent and $1.0 \% \mathrm{NS}$, and M8 containing $1.0 \%$ water-reducing agent and $1.5 \%$ NS. It should be noted that due to the low density, the NS particles are dissolved in water containing the superplasticizer and, then, gradually added to the mortar mixture. Besides, to meet the requirement of workability of cement, water consumption in the cement mixture is a little bit high: the ratio of water to cement is 0.5 . The aggregates are medium sands (less than $2.5 \mathrm{~mm}$ ), and the cement-sand ratio is 1.0 . The characteristics of the sand aggregates are presented in Table 3.

2.2. Specimen Preparation. According to the common size of mold in China, two specimen sizes are used: larger specimen size (SP-L): $150 \mathrm{~mm} \times 150 \mathrm{~mm} \times 150 \mathrm{~mm}$, reinforcement diameter: $18 \mathrm{~mm}$ (Figure 1(a)); smaller specimen size (SP-S): $50 \mathrm{~mm} \times 50 \mathrm{~mm} \times 50 \mathrm{~mm}$, reinforcement diameter: $18 \mathrm{~mm}$ (Figure $1(\mathrm{~b})$ ). Each mixture contains six specimens $(\mathrm{SP}-\mathrm{L} \times 3$ and $\mathrm{SP}-\mathrm{S} \times 3)$.

2.3. Test Procedure. The load method of single-ended drawing is used in the central pullout test, which is carried out in a WAW-1000 computer-controlled electro-hydraulic servo universal testing system. To conveniently complete the test, specimens are placed in a particular fixture, which is composed of upper and lower steel plates and four steel auxiliaries. Moreover, grating the fixture in the upper and lower ends would allow easier handling of the specimens. To avoid tearing the cement matrix caused by the skew of tensile reinforcement, there is a spherical steel hinge on the fixture plate. The test device is shown in Figure 2.

Relative slip and test load between the reinforcement and mortar are measured in the process of the central pullout test. The test method is as follows: to measure the relative slip values of the free end and the loading end of the specimen, a displacement sensor is arranged on each end. The range of the displacement sensor at the free end is $10 \mathrm{~mm}$ while the range at the loading end is $30 \mathrm{~mm}$. The sensor probes are supported on the iron, which is fixed on the surface of the specimen. The displacement sensor is connected to the data acquisition system DH3816 and collects data every $3 \mathrm{~s}$. Test load is directly read by the tester and manually recorded during the central pullout test, whereas the relative slip value is collected by the DH3816 automatically. A stress control method is used for the central pullout test during loadings, and the loading velocity is $0.1 \mathrm{kN} / \mathrm{s}$. The test is stopped when the mortar splits or the relative slip value arrives $6 \mathrm{~mm}$ at the free end.

In the following parts, the relative slip value at the free end (displacement) will be analyzed with loading stress to investigate the antipullout behaviour.

\section{Results and Discussion}

3.1. Antipullout Test Results. Figures 3 and 4 present, respectively, the relationship between loading stress and displacement with larger and smaller specimens for all eight different set of cement mixtures. Based on the results, it can be stated that the peak values of loading stresses, used as the antipullout strength, are obtained quickly with a small displacement about $1.0 \mathrm{~mm}$, and then, the loading stresses decrease gradually until a displacement of $5.5 \mathrm{~mm}$ where the substrate is completely pulled out.

The added NS can obviously increase the gripping force at a given displacement: the more the NS is added, the higher the gripping force is obtained, particularly for the specimens with $1.0 \%$ water-reducing agent.

Comparing Figures 3 and 4, the gripping force or the loading stress is higher in a specimen size of $50 \mathrm{~mm} \times 50 \mathrm{~mm} \times 50 \mathrm{~mm}$ compared to the larger size of $150 \mathrm{~mm} \times 150 \mathrm{~mm} \times 150 \mathrm{~mm}$ with the same mortar mixture. It can be explained by the constraint effect of fixture 
TABle 1: Chemical composition of cement PC32.5R.

\begin{tabular}{llllllllll}
\hline $\mathrm{CaO}$ & $\mathrm{Al}_{2} \mathrm{O}_{3}$ & $\mathrm{SiO}_{2}$ & $\mathrm{Fe}_{2} \mathrm{O}_{3}$ & $\mathrm{SO}_{3}$ & $\mathrm{MgO}$ & $\mathrm{TiO}_{2}$ & $\mathrm{~K}_{2} \mathrm{O}$ & $\mathrm{Na}_{2} \mathrm{O}$ & $\mathrm{Loss}$ \\
\hline $63.09(\%)$ & 6.59 & 20.88 & 2.73 & 2.83 & 2.09 & 0.52 & 0.09 & 0.11 & 1.07 \\
\hline
\end{tabular}

TABLE 2: Composition of the mortar mixture.

\begin{tabular}{|c|c|c|c|c|c|c|c|}
\hline \multirow{2}{*}{ Number } & \multirow{2}{*}{ Cement (kg) } & \multirow{2}{*}{ Sand $(\mathrm{kg})$} & \multirow{2}{*}{ Water $(\mathrm{kg})$} & \multicolumn{3}{|c|}{ Additive NS (g) } & \multirow{2}{*}{$1.0 \%$ water-reducing agent $(\mathrm{g})$} \\
\hline & & & & $0.5 \%$ & $1.0 \%$ & $1.5 \%$ & \\
\hline M1 & 15.1302 & 15.1302 & 7.5651 & \multirow{3}{*}{113.4765} & \multirow{4}{*}{226.953} & & \\
\hline M2 & 15.1302 & 15.1302 & 7.5651 & & & & \\
\hline M3 & 15.1302 & 15.1302 & 7.5651 & & & & \\
\hline M4 & 15.1302 & 15.1302 & 7.5651 & \multirow{5}{*}{113.4765} & & 340.4295 & \\
\hline M5 & 15.1302 & 15.1302 & 7.5651 & & & & 226.953 \\
\hline M6 & 15.1302 & 15.1302 & 7.5651 & & \multirow{3}{*}{226.953} & & 226.953 \\
\hline M7 & 15.1302 & 15.1302 & 7.5651 & & & & 226.953 \\
\hline M8 & 15.1302 & 15.1302 & 7.5651 & & & 340.4295 & 226.953 \\
\hline
\end{tabular}

TABLE 3: Characteristics of the sand aggregates.

\begin{tabular}{lllll}
\hline Size $(\mathrm{mm})$ & Fineness modulus & Water content $(\%)$ & Bulk density $\left(\mathrm{kg} / \mathrm{m}^{3}\right)$ & Silt content $(\%)$ \\
\hline$<2.5$ & 2.3 & 0.1 & 1420 & 2.5 \\
\hline
\end{tabular}

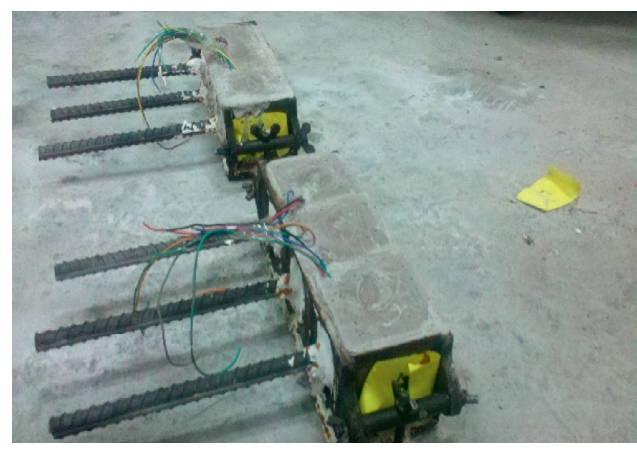

(a)

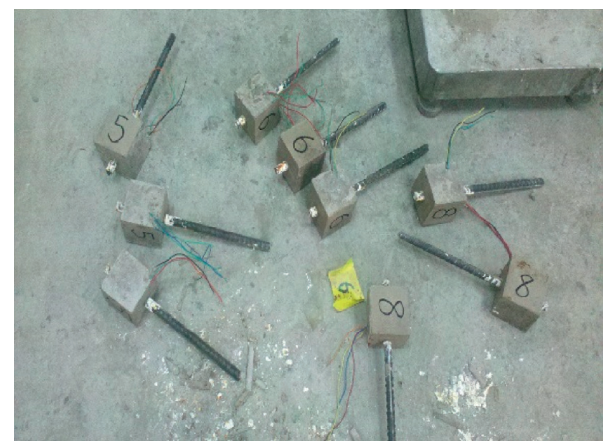

(b)

Figure 1: Mortar specimens with different sizes: (a) SP-L; (b) SP-S.

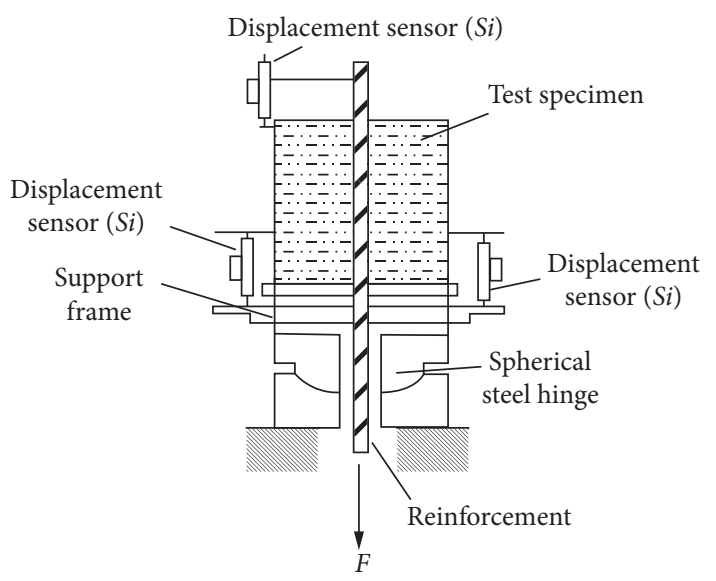

Figure 2: Central pullout test device. to the smaller size specimens. The increase of loading stress is almost the same for two specimen sizes as increasing NS.

3.2. Antipullout Strength. As mentioned above, the peak value of loading stress is used as the antipullout strength in this study.

Figure 5 shows the variation of the antipullout strength with added NS for two different sizes. The results indicate that the antipullout strength decreases at least 15\% from a smaller size specimen to a larger size specimen.

Besides, the antipullout strength increases with the NS content: with $1.0 \%$ water-reducing agent, the antipullout strength increases at most 33\% with an increase in the NS content from 0 to $1.0 \%$. Without the water-reducing agent, the increase in the antipullout strength is about $60 \%$ with an increase in the NS content from 0 to $1.5 \%$.

Figure 6 shows the variation of the antipullout strength with added NS with and without the water-reducing agent. It 


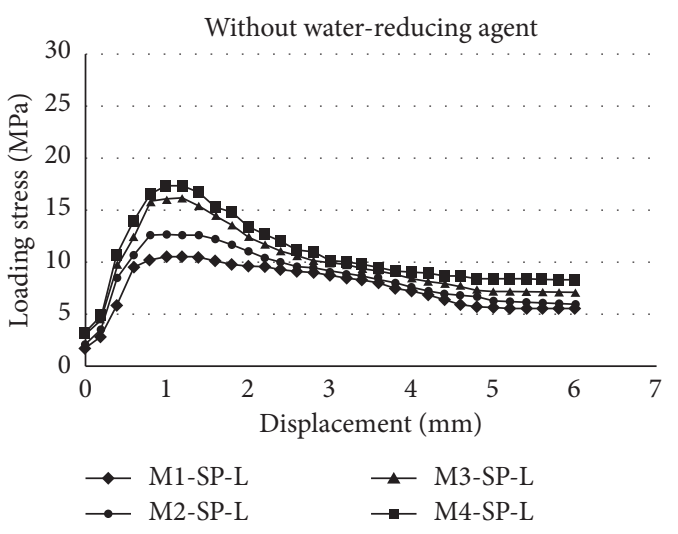

(a)

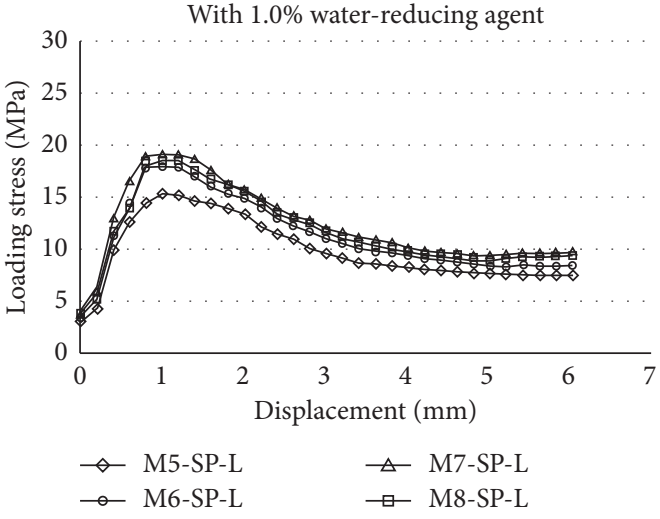

(b)

Figure 3: Relationship between loading stress and displacement with larger specimens: (a) without the water-reducing agent; (b) with 1.0\% water-reducing agent.

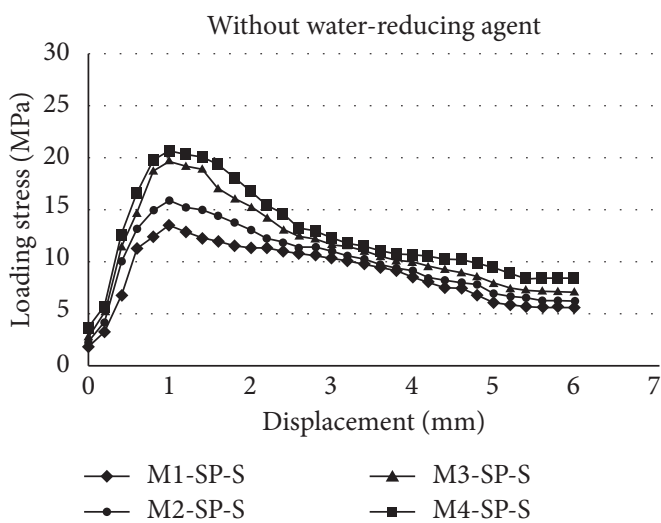

(a)

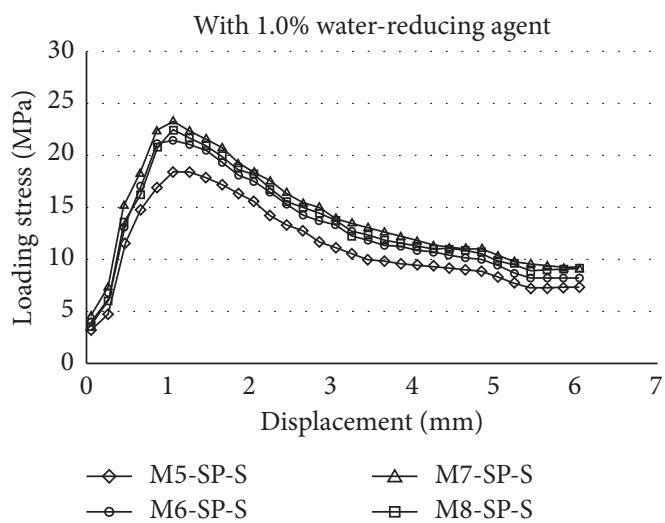

(b)

FIGURE 4: Relationship between loading stress and displacement with smaller specimens: (a) without the water-reducing agent; (b) with 1.0\% water-reducing agent.

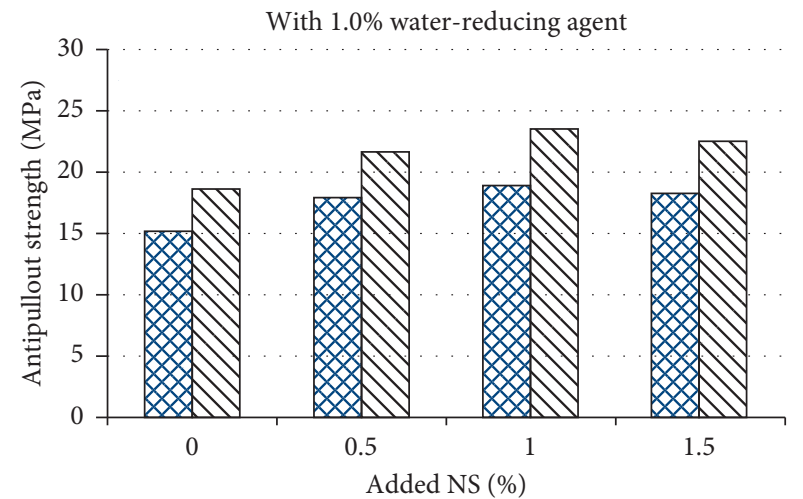

SP-L

$\triangle$ SP-S

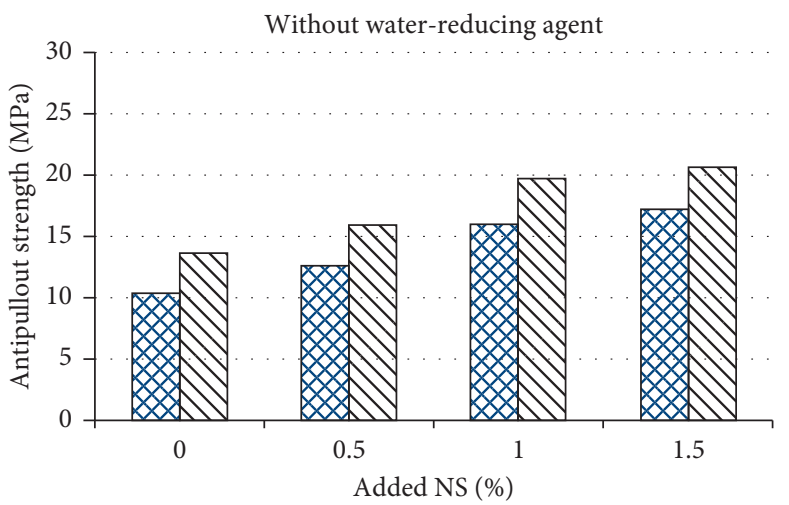

BSP-L

$\triangle$ SP-S

(a)

(b)

FIGURE 5: Variation of the antipullout strength with added NS for two different sizes: (a) with 1.0\% water-reducing agent; (b) without the water-reducing agent. 


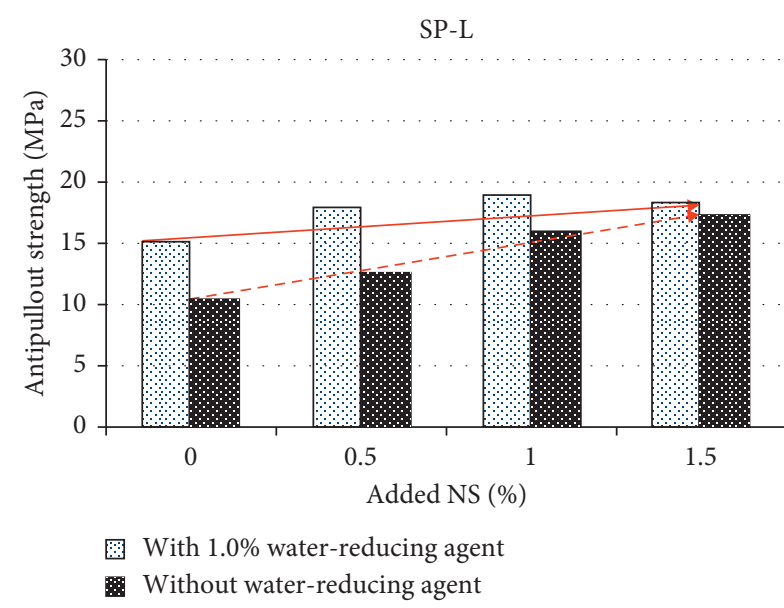

(a)

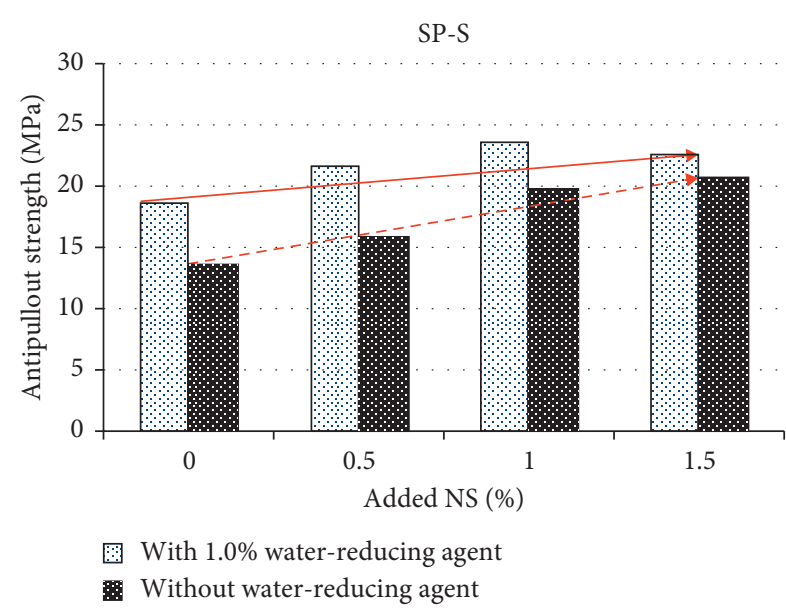

(b)

FIGURE 6: Variation of the antipullout strength with added NS with or without the water-reducing agent: (a) larger specimen size; (b) smaller specimen size.

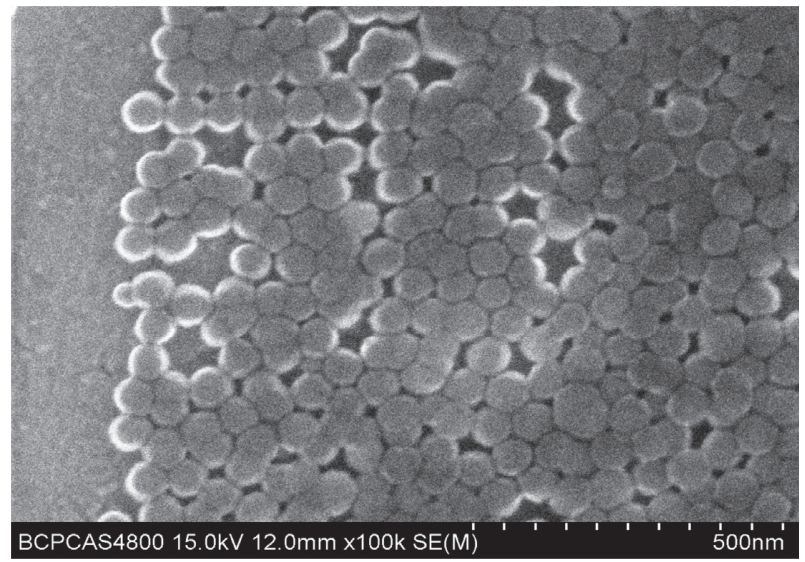

FIGURE 7: SEM image of the NS.

can be stated that the incorporation of a water-reducing agent can increase the antipullout strength with the same content of added NS. The increase in the antipullout strength caused by the water-reducing agent is getting weaker with an increasing NS content and the increase disappears, especially when the NS content is about $1.5 \%$.

Overall, the antipullout strength is the lowest (10.5 MPa for a larger sample and 13.5 MPa for a smaller sample) for the mixture without any additive. The antipullout strength is the largest (19 MPa for a larger sample and $23.5 \mathrm{MPa}$ for a smaller sample) for the mixture with $1.0 \%$ NS and $1.0 \%$ water-reducing agent.

The results indicate that the gripping force between the reinforcement and mortar can be increased sharply by adding a certain content of NS and the water-reducing agent; thus, the properties of reinforcement and cement can be fully utilized.

\subsection{Effect Mechanism}

3.3.1. Theoretical Analysis. In the initial cement hydration period, the reaction mainly occurs on the surface of cement particles forming hydration products without any additives. The hydration products continuously deposit on the surface of the cement particles during the reaction. Exchanging the ions between unhydrated cement particles and the external solution, then, becomes difficult, thereby preventing the hydration reaction and the formation of Calcium Silicate Hydrate (CSH) [25].

Generally, the performances of strength and durability of cement materials depend on the content of $\mathrm{CSH}$ and the porosity of the cement structure. The performance can be improved from the following aspects: (1) The porosity of the cement structure can be reduced by the incorporation of NS. (2) The CSH content can be increased by the reaction of NS and Calcium Hydroxide $(\mathrm{CH})$, filling the space between the cement particles and making the microstructure more close-grained to improve the performance of the hardened cement paste [26-28]. (3) The speed of hydration reaction can be promoted [29], whereas the NS is in the induction of hydration products, thereby promoting the growth of $\mathrm{CSH}$ gel and filling the gaps between aggregates. (4) As the high activity, NS can react with calcium hydroxide producing secondary hydration products and reducing the porosity of the cement mortar. Therefore, the mechanical properties and the friction force between reinforcement and the hardened cement paste can be improved, and then, the antipullout capacity increases $[12,13]$. (5) Besides, silica fume size distribution is a key factor regarding the compressive strength development. The silica fume with high fineness is a suitable pozzolanic material to be used in producing a better high-performance concrete. The addition of fine silica fume to Portland cements has been shown to give rise to physical (i.e., filler) and chemical (i.e., pozzolanic) effects on the microstructure of hardened pastes, leading to improved macroproperties of mortars and concretes, such as higher strength and lower permeability, among others [30]. 


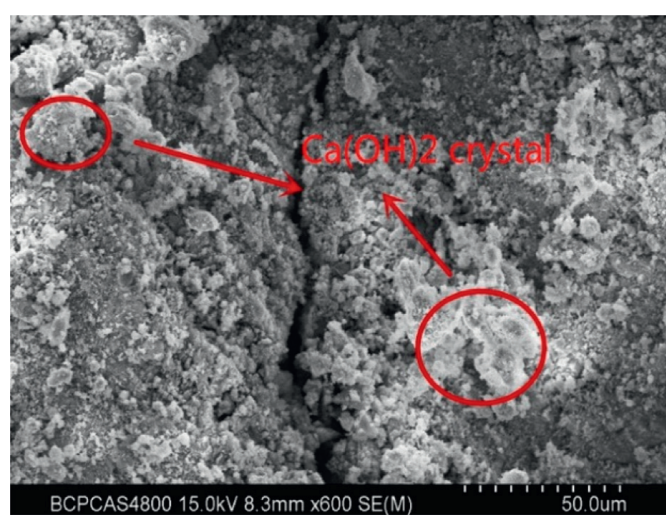

(a)

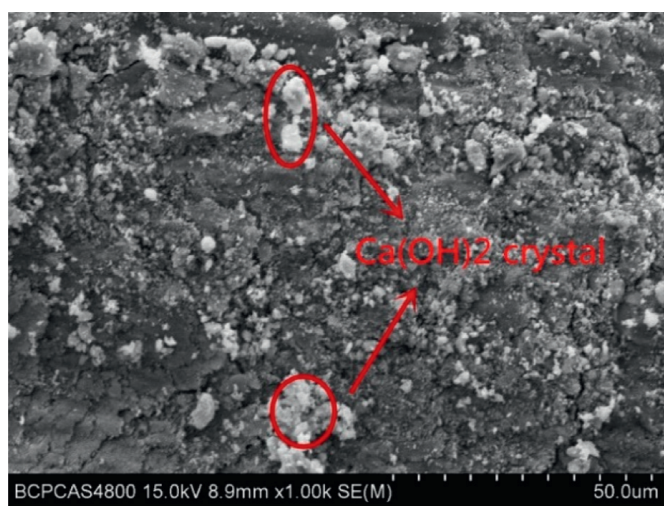

(c)

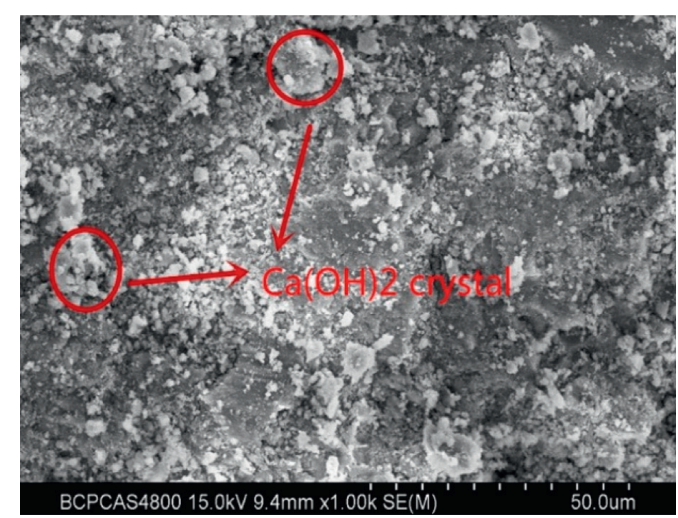

(b)

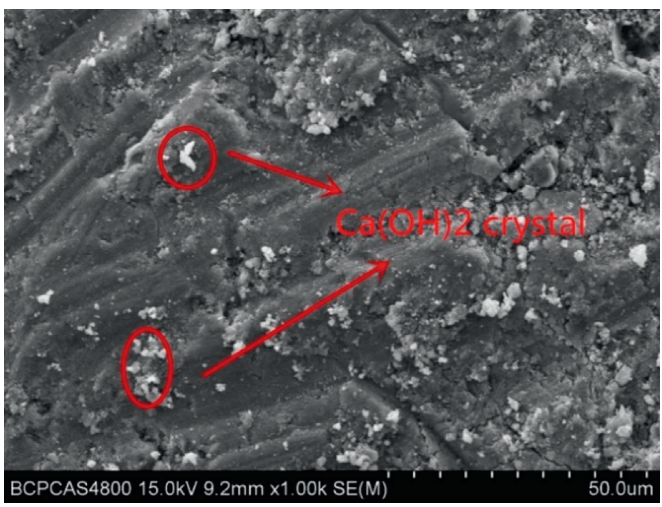

(d)

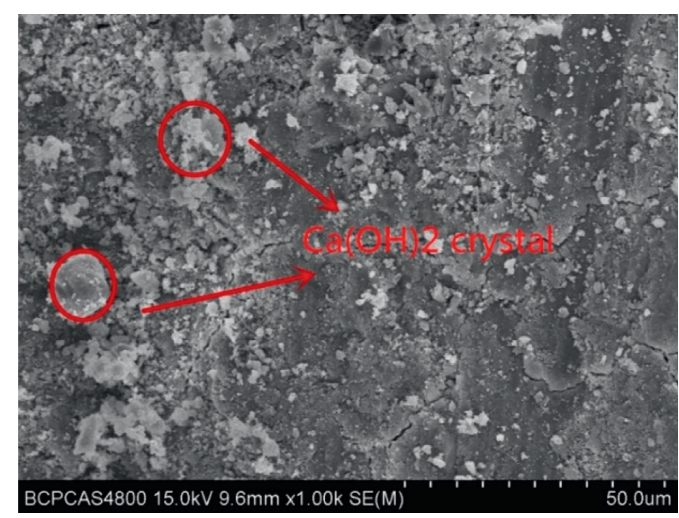

(e)

FIGURE 8: SEM images of cement mortar specimens with different blending proportions: (a) no additive, (b) $1.0 \%$ water-reducing agent, (c) $0.5 \% \mathrm{NS}+1.0 \%$ water-reducing agent, (d) $1.0 \% \mathrm{NS}+1.0 \%$ water-reducing agent, and (e) $1.5 \% \mathrm{NS}+1.0 \%$ water-reducing agent.

In the following part, the calcium hydroxide $\left(\mathrm{Ca}(\mathrm{OH})_{2}\right)$ in the hardened cement paste with different cement mixture will be investigated by SEM to validate the effects of additives (NS and water-reducing agent).

3.3.2. Microscopic Analysis. SEM images of NS are obtained firstly with a Hitachi S-4800 field emission scanning electron microscope (http://www.hitachi-hightech.com) using the aqueous dispersion of NS placed on the SEM specimen support (aluminium), subsequently evaporated at room temperature and coated with Au. After the pullout test, the center section of the broken specimen is rubbed to the size of
$8 \mathrm{~mm} \times 8 \mathrm{~mm} \times 1 \mathrm{~mm}$, and the microstructure is, then, immediately investigated by SEM.

Figure 7 shows the SEM image of the NS. It can be observed that the size of the prepared NS particle is about $500 \mathrm{~nm}$ in diameter. The high fineness of the NS is a key factor regarding the mortar compressive strength development, which can give rise to physical and chemical effects on the microstructure of hardened pastes [30].

Figure 8 shows the microstructures of cement mortar specimens with different mixture proportions observed in SEM. It is found that CSH in the microstructure of cement mortar specimens without NS is loosely arranged, whose surface existed a lot of $\mathrm{Ca}(\mathrm{OH})_{2}$ crystals. In particular, 
Figure 8 (a) presents that the content of $\mathrm{Ca}(\mathrm{OH})_{2}$ in a specimen with no additives is significantly higher than that in the following four specimens with NS and the waterreducing agent. The content of $\mathrm{Ca}(\mathrm{OH})_{2}$ shown in Figure $4(\mathrm{~b})$ is reduced by about $10 \%$ compared to Figure 4(a). This result indicates that the water-reducing agent plays a certain promoting role in the formation of $\mathrm{CSH}$.

Figures $8(\mathrm{c})-8(\mathrm{e})$ show, respectively, the microstructures as the NS content increases (from $0.5 \%$ to $1.5 \%$ ) with $1.0 \%$ water-reducing agent. Based on the results, it can be stated that the content of $\mathrm{Ca}(\mathrm{OH})_{2}$ and the mortar porosity decrease gradually with an increase in the NS content from 0 to $1.0 \%$. The very few $\mathrm{Ca}(\mathrm{OH})_{2}$ crystals can be founded when the content of added NS is about $1 \%$.

However, increasing NS content to $1.5 \%$, the $\mathrm{Ca}(\mathrm{OH})_{2}$ crystals become obvious again, as shown in Figure 8(e). It can be explained as the viscosity of the mortar increases with the addition of NS and the locked air can prevent the hydration reaction. Thus, the content of $\mathrm{Ca}(\mathrm{OH})_{2}$ crystal increases.

Generally, the SEM results confirm that

(1) The water-reducing agent can promote the formation of $\mathrm{CSH}$.

(2) By promoting the dissolution and self-hydration of cement particles to generate granular hydration products, which are cement hydration crystal nucleus, NS can promote the crystallization of hydration products, thereby promoting cement hydration $[15,31]$.

(3) A moderate amount of NS (1.0\%) can improve the microstructure and macroscopic properties of cement to have more superior mechanical properties and enhanced durability. It can also validate and explain the antipullout test results.

\section{Conclusions and Discussion}

The effects of nanoparticles, the water-reducing agent, and the specimen size on the antipullout strength between the reinforcement and cement mortar are evaluated in this study. Based on the discussion above, the following primary conclusions can be obtained:

(1) The gripping force is larger with a smaller specimen size, and it can be explained by constraint effect of fixtures. There is no obvious effect of specimen size on the mechanism of incorporation of NS.

(2) The water-reducing agent can promote the formation of $\mathrm{CSH}$ and, thus, increase the antipullout strength.

(3) The cement antipullout strength can be improved by the incorporation of NS and achieves the largest value with an optimal content of NS (1.0\%). The microscopic analysis with SEM also validates this result: NS can accelerate the hydration reaction of cement, produce more CSH gel, and increase the density of the cement mortar, thereby improving the microstructure and macroscopic mechanical properties. With the optimal content of NS (1.0\%), specimen has the most close-grained structure, and very few $\mathrm{Ca}(\mathrm{OH})_{2}$ crystals can be founded, thereby leading to the highest antipullout strength.

These results would provide guidance on the design or construction of a cement mortar with reinforcement.

\section{Data Availability}

The data used to support the findings of this study are included within the article.

\section{Conflicts of Interest}

The authors declare that there are no conflicts of interest regarding the publication of this paper.

\section{Acknowledgments}

The authors acknowledge the support from the National Key R\&D Program of China under grant no. 2017YFC0805305, the Science and Technology Development Fund of Macao China (FDCT) under grant no. 0010/2018/A, the National Natural Science Foundation (NSFC) under grant no. 41772330, and the Scientific Research and Development Program of China Railway under grant P2018G004.

\section{References}

[1] W. H. Baker and R. L. Konder, "Pullout load capacity of a circular earth anchor buried in sand," Highway Research Record, vol. 108, pp. 1-10, 1966.

[2] E. A. Dickin and C. F. Leung, "Centrifugal model tests on vertical anchor plates," Journal of Geotechnical Engineering, vol. 109, no. 12, pp. 1503-1525, 1983.

[3] F. A. Barber, P. Cawley, and J. F. Prudich, "Suture anchor failure strength-An in vivo study," Arthroscopy: The Journal of Arthroscopic \& Related Surgery, vol. 9, no. 6, pp. 647-652, 1993.

[4] Y. C. Flores, G. C. Cordeiro, R. D. Toledo Filho, and L. M. Tavares, "Performance of Portland cement pastes containing nano-silica and different types of silica," Construction and Building Materials, vol. 146, pp. 524-530, 2017.

[5] H. F. W. Taylor, "Nanostructure of CSH: current status," Advanced Cement Based Materials, vol. 1, no. 1, pp. 38-46, 1993.

[6] S. Mann, "Nanotechnology and construction," p. 55, 2006 Nanoforum Report.

[7] K. Sobolev and M. F. Gutiérrez, "How nanotechnology can change the concrete world," American Ceramic Society Bulletin, vol. 84, no. 11, pp. 113-116, 2005.

[8] B. H. Green, "Development of a high-density cementitious rock-matching grout using nano-particles," ACI Special Publication, vol. 254, pp. 121-132, 2008.

[9] G. Quercia and H. Brouwers, "Application of nano-silica (NS) in concrete mixtures," in Proceedings of the 8th Fib PhD Symposium in Civil Engineering, Lyngby, Denmark, June 2010, http://www.researchgate.net/publication/257029738_ Application_of_nano-silica_nS_in_concrete_mixtures.

[10] L. Senff, D. Hotza, W. L. Repette, V. M. Ferreira, and J. A. Labrincha, "Mortars with nano-Sio ${ }_{2}$ and micro-Sio ${ }_{2}$ 
investigated by experimental design," Construction and Building Materials, vol. 24, no. 8, pp. 1432-1437, 2010.

[11] T. Ji, "Preliminary study on the water permeability and microstructure of concrete incorporating nano-Sio ${ }_{2}$," Cement and Concrete Research, vol. 35, no. 10, pp. 1943-1947, 2005.

[12] M. Bastami, M. Baghbadrani, and F. Aslani, "Performance of nano-silica modified high strength concrete at elevated temperatures," Construction and Building Materials, vol. 68, pp. 402-408, 2014.

[13] R. Yu, P. Spiesz, and H. J. H. Brouwers, "Effect of nano-silica on the hydration and microstructure development of ultrahigh performance concrete (UHPC) with a low binder amount," Construction and Building Materials, vol. 65, pp. 140-150, 2014.

[14] R. Yu, P. Spiesz, and H. J. H. Brouwers, "Mix design and properties assessment of ultra-high performance fibre reinforced concrete (UHPFRC)," Cement and Concrete Research, vol. 56, pp. 29-39, 2014.

[15] R. Yu, P. Tang, P. Spiesz, and H. J. H. Brouwers, "A study of multiple effects of nano-silica and hybrid fibres on the properties of ultra-high performance fibre reinforced concrete (UHPFRC) incorporating waste bottom ash (WBA)," Construction and Building Materials, vol. 60, pp. 98-110, 2014.

[16] Q. Ye, Z. Zhang, D. Kong, R. Chen, and C. Ma, "Comparison of properties of high-strength concrete with nano-Sio ${ }_{2}$ and silica fume added," Journal of Building Materials, vol. 6, no. 4, pp. 281-285, 2003.

[17] H. Li, H.-g. Xiao, J. Yuan, and J. Ou, "Microstructure of cement mortar with nano-particles," Composites Part B: Engineering, vol. 35, no. 2, pp. 185-189, 2004.

[18] S. Hanehara and K. Yamada, "Interaction between cement and chemical admixture from the point of cement hydration, absorption behaviour of admixture, and paste rheology," Cement and Concrete Research, vol. 29, no. 8, pp. 1159-1165, 1999.

[19] Ş. Erdoğdu, "Compatibility of superplasticizers with cements different in composition," Cement and Concrete Research, vol. 30 , no. 5, pp. 767-773, 2000.

[20] L. Raki, J. Beaudoin, R. Alizadeh, J. Makar, and T. Sato, "Cement and concrete nanoscience and nanotechnology," Materials, vol. 3, no. 2, pp. 918-942, 2010.

[21] A. Sadrmomtazi and A. Barzegar, "Assessment of the effect of nano-Sio ${ }_{2}$ on physical and mechanical properties of selfcompacting concrete containing rice husk ash," in Proceedings of the Second International Conference on Sustainable Construction Materials and Technologies, Universita Politecnica delle Marche, London, UK, June 2010.

[22] A. Nazari and S. Riahi, "The effects of $\mathrm{Sio}_{2}$ nanoparticles on physical and mechanical properties of high strength compacting concrete," Composites Part B: Engineering, vol. 42, no. 3, pp. 570-578, 2011.

[23] K. Wang and G. R. Lomboy, "Nanoparticle modified concrete materials: opportunities, challenges and prosperities, construction materials and structures," in Proceedings of the First International Conference on Construction Materials and Structures, pp. 16-26, Johannesburg, South Africa, November 2014.

[24] L. P. Singh, A. Goel, S. K. Bhattachharyya, S. Ahalawat, U. Sharma, and G. Mishra, "Effect of morphology and dispersibility of silica nanoparticles on the mechanical behaviour of cement mortar," International Journal of Concrete Structures and Materials, vol. 9, no. 2, pp. 207-217, 2015.
[25] R. Krstulović and P. Dabić, "A conceptual model of the cement hydration process," Cement and Concrete Research, vol. 30, no. 5, pp. 693-698, 2000.

[26] B.-W. Jo, C.-H. Kim, G.-h. Tae, and J.-B. Park, "Characteristics of cement mortar with nano-Sio 2 particles," Construction and Building Materials, vol. 21, no. 6, pp. 1351-1355, 2007.

[27] N. León, J. Massana, F. Alonso, A. Moragues, and E. SánchezEspinosa, "Effect of nano- $\mathrm{Si}_{2} \mathrm{O}$ and nano- $\mathrm{Al}_{2} \mathrm{O}_{3}$ on cement mortars for use in agriculture and livestock production," Biosystems Engineering, vol. 123, pp. 1-11, 2014.

[28] P. Hou, X. Cheng, J. Qian, R. Zhang, W. Cao, and S. P. Shah, "Characteristics of surface-treatment of nano-SiO2 on the transport properties of hardened cement pastes with different water-to-cement ratios," Cement and Concrete Composites, vol. 55, pp. 26-33, 2015.

[29] H. Sattawat, P. Tawich, and C. Somchai, "Effect of nanosilica particle size on the water permeability, abrasion resistance, drying shrinkage, and repair work properties of cement mortar containing nano- $\mathrm{SiO}_{2}$," Advances in Materials Science and Engineering, vol. 2017, Article ID 4213690, 11 pages, 2017.

[30] M. Á. Sanjuán, C. Argiz, J. C. Gálvez, and A. Moragues, "Effect of silica fume fineness on the improvement of Portland cement strength performance," Construction and Building Materials, vol. 96, pp. 55-64, 2015.

[31] A. Kumar, T. Oey, S. Kim et al., "Simple methods to estimate the influence of limestone fillers on reaction and property evolution in cementitious materials," Cement and Concrete Composites, vol. 42, pp. 20-29, 2013. 